\title{
Large scale nucleosynthetic heterogeneities across the solar system identified by xenon
}

\author{
G. AvicE ${ }^{1}$, J. D. GILMOUR ${ }^{2}$, M. MOREIRA ${ }^{1}$ \\ ${ }^{1}$ Université de Paris, Institut de physique du globe de Paris, \\ CNRS, F-75005 Paris, France. \\ ${ }^{2}$ Dept of Earth and Environmental Sciences, School of \\ Natural Sciences, University of Manchester, UK.
}

The formation of the solar system involved large-scale mixing of material throughout the entire protoplanetary disk. However, studies demonstrated the persistence of two distinct isotopic reservoirs in the solar system. Carbonaceous chondritic material, from the outer regions of the solar system, carries excesses of neutron-rich $\mathrm{Cr}$, $\mathrm{Ti}, \mathrm{Mo}, \mathrm{Ni}$ isotopes compared to non-carbonaceous material [1-3]. The origin of this dichotomy remains debated e.g. [1-5].

Noble gases are historical tracers of choice for understanding nucleosynthetic heterogeneities in solar system material. Xenon is particularly useful since its nine isotopes $\left({ }^{124-136} \mathrm{Xe}\right)$ are produced by $\mathrm{P}-, \mathrm{R}-$ and $\mathrm{S}$-process stellar nucleosynthesis. Studying the relative proportions of $\mathrm{Xe}$ isotopes in solar system material contributes to put constraints on the different nucleosynthetic environments $[6$ \& refs. therein] and to establish their influences on the distribution of nuclides in different regions of the solar system.

In this study [7], we show that the xenon data from comet 67P/Churyumov-Gerasimenko [8] can be re-interpreted with the theoretical framework developed in previous studies of nucleosynthetic anomalies in meteorites [9]. Results are used to compute the isotopic composition of the theoretical starting component for Earth's atmospheric xenon, U-Xe [10], a mixture of cometary $(\sim 20 \%)$ and chondritic $(\sim 80 \%)$ Xe [8]. The isotopic composition of this component demonstrates the existence of a temporal or physical separation establishing relative variations of $\mathrm{P}$-process and $\mathrm{R}$-process $\mathrm{Xe}$ isotopes across the Solar system. Either the incorporation of P-process happened before incorporation of R-process nuclides or material in the outer edge of the solar system carries a different mix of presolar sources as in parent bodies of meteorites.

[1] Trinquier et al. (2009) Science, 324, 374. [2] Warren (2011) EPSL, 311, 93-100. [3] Kruijer et al. (2017) PNAS, 312, 201704461-15. [4] Ek et al. (2019) Nat. Astron., 4, 273. [5] Jacquet et al. (2019) ApJ, 884, 32. [6] Ott U. (2002) RiMG, 47, 71-100. [7] Avice et al. (2020) ApJ, 889, 68. [8] Marty et al. (2017) Science, 356, 1069-1072. [9] Gilmour \& Turner (2007) ApJ, 657, 600-608. [10] Pepin (1991) Icarus, 92, 2 . 